\title{
Research on the Innovative Design of Chinese Characters by Deconstruction
}

\author{
Jing Hou \\ Zaozhuang University \\ Zaozhuang, China
}

\begin{abstract}
This study takes the regular script as an example. The author breaks down the spatial structure of Chinese characters by deconstruction, and forms a new font style to explore the possibility of Chinese character font design in the current era. Chinese writing is a formal system consisting of five levels from simplicity to complication: elements - individual strokes; modules - construction or components; units - individual Chinese characters; series - coherent text; mass - all Chinese characters. In this paper, the deconstructionist approach is introduced, in which the strokes (elements) of Chinese characters remain unchanged. Through the dispersal of the original structure of the characters and the establishment of different reorganization modes, multiple font forms of individual Chinese characters are created, and then a sequence or collection will be formed by choosing the combination modes with other Chinese characters, so as to meet the deep needs of users who hope to choose the ideal combination of fonts independently.
\end{abstract}

Keywords-Chinese characters; deconstructionist approach; font design; innovative design

\section{INTRODUCTION}

Font design is the most important cornerstone of visual communication design. Whether it is logo design, book design, web design, brand image design or information design, fonts are indispensable. But as far as China's current situation is concerned, font design is still neglected. There are some problems in the spread and application of Chinese characters, such as less font selection, poor font quality and uniformity. Conservative design, lack of novelty, shortage of design personnel and other unfavorable factors make the domestic font design unable to step out of the predicament of overall slow development.

\section{FORMAL LANGUAGE AND EXPRESSIVE CHARACTERISTICS OF DECONSTRUCTIONISM}

Deconstruction is translated into deconstructionism in the field of design, while it is translated as "decomposition criticism" or "decomposition theory" in the field of philosophy and linguistics. Literally, the word "Jie" means "decompose" and "Gou" means "structure and composition". So, the word "deconstruction" can be extended to "decompose and then form". In the new exploration of formal language, deconstructionism is essentially the destruction and decomposition of structuralism, advocating dissolution, separation, fragmentation, incompleteness and no center. In terms of design, deconstructionism is not anarchism. All deconstructionist works are seemingly chaotic, but in essence, they have highly theoretical characteristics of internal structural factors and overall consideration.

The diversification of deconstructive form features is determined by the diversity of its design methods and design perspectives. Although deconstructionism is applied in different fields and its methods vary widely, the common point is to express the features of "nothing", "non-functional", "irrational" and "anti-form" by "decomposing" and "breaking". In the field of art and vision, many people believe that deconstructionism can exist as a formal language or even a modeling method. "I think the problem with deconstruction is that it becomes a style", ${ }^{1}$ Eisenman said. When discussing whether a designer uses deconstruction techniques or how high the quality and level of deconstruction works are, it needs to be judged by the visual language of the design works. In his book Architecture and Deconstruction, Professor Wu Huanjia sums up some common forms and characteristics of deconstruction architecture-based works that are generally accepted: scattered, incomplete, suddenly changed, empowered, extraordinary and so on. Some researchers have proposed that "if there are more and more designs with specific image characteristics, and these formal languages can ensure relative stability in a certain period of time, a kind of style will be formed." Deconstruction masters in various fields have launched deconstruction works, which aroused public imitation and reference, thus becoming a fashion.

\section{DeCOnstruction Methods OF ChInESE Fonts}

\section{A. Font Focus Shift}

Modern font design research pays full attention to the use of modern art and science principles and aesthetic principles to explain many problems in design process. Many writings or books have repeatedly emphasized that the focus of fonts should be on upper location, which can be explained by the principle of artistic visual illusion. Comparing the absolute "geometric center" with the "visual center" of the human eye test, the difference between the two can be found out, and the scientificity of the comparison in a mathematical way will be demonstrated. Thus, a conclusion will be draw that the "visual center" of human beings lies in one-second of the square center

Wu Lieyan writes: Deconstructive Design, Jiangsu Fine Arts Publishing House, August 2001, P. 95 
and the top line of $\sqrt{3}$ rectangles. ${ }^{2}$ Through the first central line and the second central line, the position of the focus center of the single character and the reasonable character is determined, so that the size of the text is basically consistent with the unified style of the whole adverb. As a standard typeface style, the standard style of handwriting is featured by stable focus, scientific design and numerical value. The focus of the font is basically located on the vertical line of the structure. If the vertical lines in the structure of these typefaces with stable center of focus are offset, they will form a structure with upper center of focus, right center of focus, left center of focus, lower center of focus, upper left center of focus, upper right center of focus, lower left center of focus and lower right of center of focus, as shown in "Fig.1". For example, for "ᄏ", the center of the printed block letters on the left falls in the center of the square box. In order to shift the center of focus to the left, the two strokes of "point" and "lift" are moved to the left, and the spatial relationship between the two strokes is changed, which makes the shape of "习" more graphical and metaphorical. In order to shift its center of focus upward, the word "书" adjusts the vertical drawing upward and slightly shifts to the left, making its new spatial structure more fragmented. Similarly," 为" has also done the corresponding experiments with the center of focus shifting to the upper left, the lower left, the upper right and the lower right, so that a standard handwriting can be transformed into different kinds of font styles with new spatial structure.

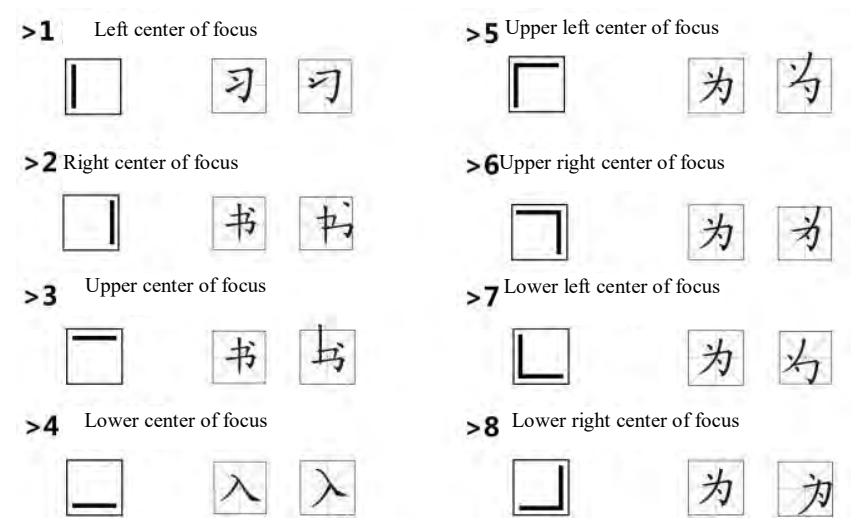

Fig. 1. Experiments on eight forms of focus center shift of regular script.

\section{B. Framework of Deconstructive Characters}

Stroke is the most basic element of Chinese characters and these strokes are connected, separated and intersected in the construction of characters. Component (module) is a characterbuilding unit composed of strokes with the function of matching Chinese characters. There are also three kinds of combinational relations between components, namely, connection, separation and intersection. Similarly, there is still a connection, separation and intersection between strokes and components. In the structure of Chinese characters, these forms are fixed. The author tries to transform the conventional way of composition between strokes and components, i.e. from separation to connection or intersection, from connection to separation or intersection, from intersection to connection or separation, etc., to shape a new way of composition of Chinese characters and form a new spatial structure relationship, as shown in "Fig.2". For example, for the word "习", it is separated from the "point", "lift" and the semi-enclosed" cross break hook ". It connects the stroke "lift" with the "cross break hook", and intentionally approaches the "point" to the "lift", creating a graphical symbolic language, and so does the character "风". For Chinese characters "入", the relationship between "left-falling" and "right-falling" itself is passive connection. By separating their strokes, they are transformed into separate forms, and meanwhile, their strokes are also intersected into intersecting forms. However, no matter how the three forms are transformed, it is not allowed to exceed the recognition degree of the Chinese characters themselves. According to this method, a series of innovative font styles can be obtained by deconstructing and transforming the formations of regular script.

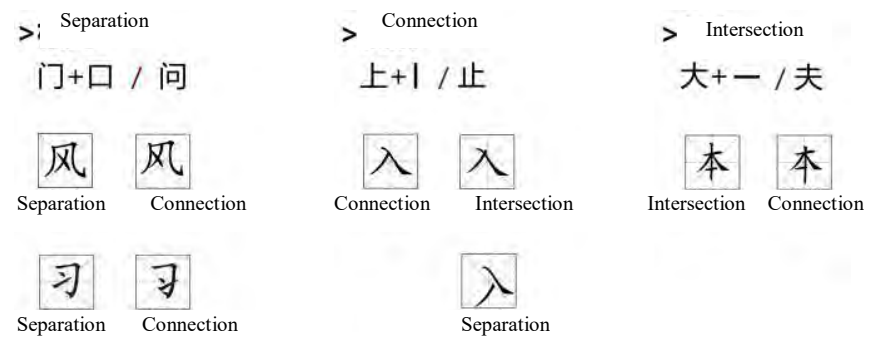

Fig. 2. Three frameworks of deconstructive standard style of handwriting.

\section{Configuration Level of Deconstructive Standard Style of Handwriting}

As a form of hierarchical structure in the process of Chinese character formation, the configuration level of Chinese characters is based on components, so it is for the multiplecomponent character. In standard typefaces, the formational level of Chinese characters does not alter depend on the change of typefaces, and its spatial position and structure are relatively fixed. For example, the character "放(fang)" is a standard structure of the combination of left and right parts. If the original structure level is broken up, through the movement of stroke position, it will form a hierarchy with blurred boundaries, i.e. the form of single character or the structure of multiple components, as shown in "Fig.3". This is an overall change in the level of configuration. Another form is to adjust a part of Chinese characters. In this condition, the level of configuration itself has not changed, but the content of the level has altered. For example, the word "场 (ao)" is composed of three parts: "土 (tu)", "幺(yao)" and "力(li)". The intermediate part of "幺(yao)" is divided and changed so that one part of it belongs to "土" and the other part belongs to "力 (li)". At this time, the font form is in the state of "betweenness". So that it can be said that it is the structural mode of two parts or three parts. This deconstruction method makes the Chinese characters present a state of unclear generic boundaries and ambiguous nature.

\footnotetext{
2 Liu Zanai: Visual Design and Fuzziness of Fine Arts Characters,
}

Decoration, Issue 2, 1986 


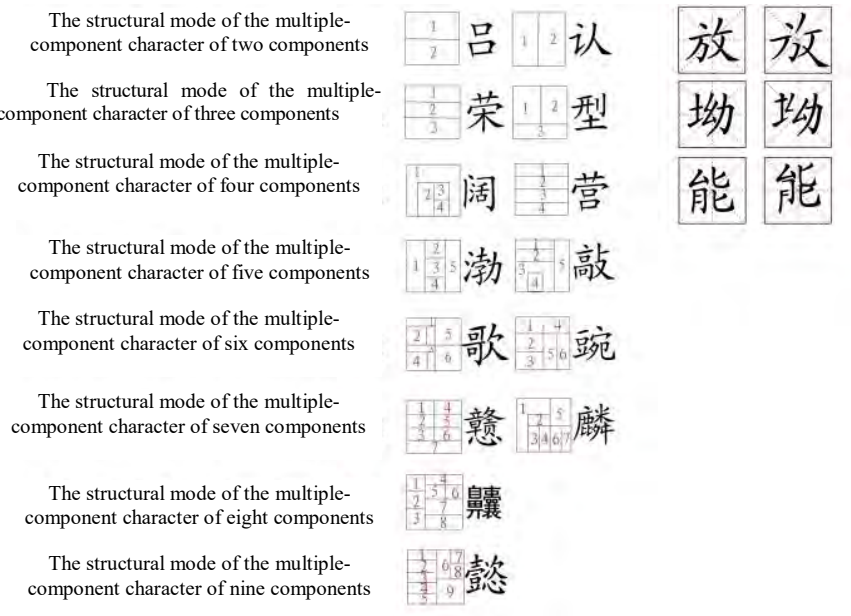

Fig. 3. Nine configuration levels of deconstructive standard style of handwriting.

\section{Deconstruction of the Combination Mode of Chinese Characters}

The "up-down structure", "parallel structure", "surround structure" and others often talked about by people refer to the combination mode of Chinese characters, and they also called the matrix structure. It can be subdivided according to the size of each structural component, for example, the left-right structure can be divided into "equal", "left wide and right narrow", "left narrow and right wide" structure; the up-down structure is divided into "equal for upper and lower" and "unequal for upper and lower". This kind of fine division provides conveniences for the deconstruction of Chinese character in classification." For the Chinese characters with equal left and right space in left-right structure, we can move the space of strokes to change the left wide and right narrow font or left narrow and right wide font; similarly, the left wide and right narrow fonts can be changed into left and equal right and left fonts or left narrow and right wide fonts, as shown in "Fig. 4". The Chinese word "耐(nai)" in the figure originally is left wide and right, and after the author adjusts the position of some strokes, the word becomes a equal left and right font and a left narrow and right wide font. Such adjustment is not unique but rich, so a standard printed font can has a series of font style with new spatial order through this deconstruction method. The triangular structure, also known as the “品(pin)” structure, is characterized by a triangular shape of the entire font with a narrow top and a wide bottom, for example, the word "轰(hong)", is a font of typical triangular structure; in addition to adjusting the position of the strokes and parts to make the word out of the triangular structure, the author also tries to deliberately enlarge the characteristics of this structure, to create a visual form of conflict, exaggeration, imbalance and discordance. The half-surrounded structure can also be subdivided into "upper surrounding lower", "lower surrounding upper", "left surrounding right", and "right surrounding left". The word "风(feng)" has a structure of "upper surrounding lower", while the author tries to open the upper bounding box to make the closed part inside out of the outer frame, forming a fuzzy polysemy combination mode, and giving the standard font form a lively and talking emotional color. In addition to some generally known combination modes, some Chinese characters seemingly in a independent structure also have their own combination mode, including superposition or conglutination, such as "更(geng)", that is the conglutination of "丙(bing)" and "支(zhi)". But here it is still deconstructed as a single character. Generally, due to physiological illusions, the right part takes a relatively larger proportion than the lower half of the structure; the larger right part mostly is caused by restraining the left and spreading the right, and the larger lower part caused by the human visual center higher than the geometric center. Usually, we say a part of a word takes onehalf or one-third in the proportion that is formed in the longterm development of Chinese characters, and this is a concept that people have established and it has certain stability. After deconstructing the combination mode of Chinese character, the font no longer follows this proportional relationship, and creates a new appreciation standard and aesthetic style, and this style has a uniform proportional relationship with the overall style of the deconstructed font. 
Matrix structure (combination mode)

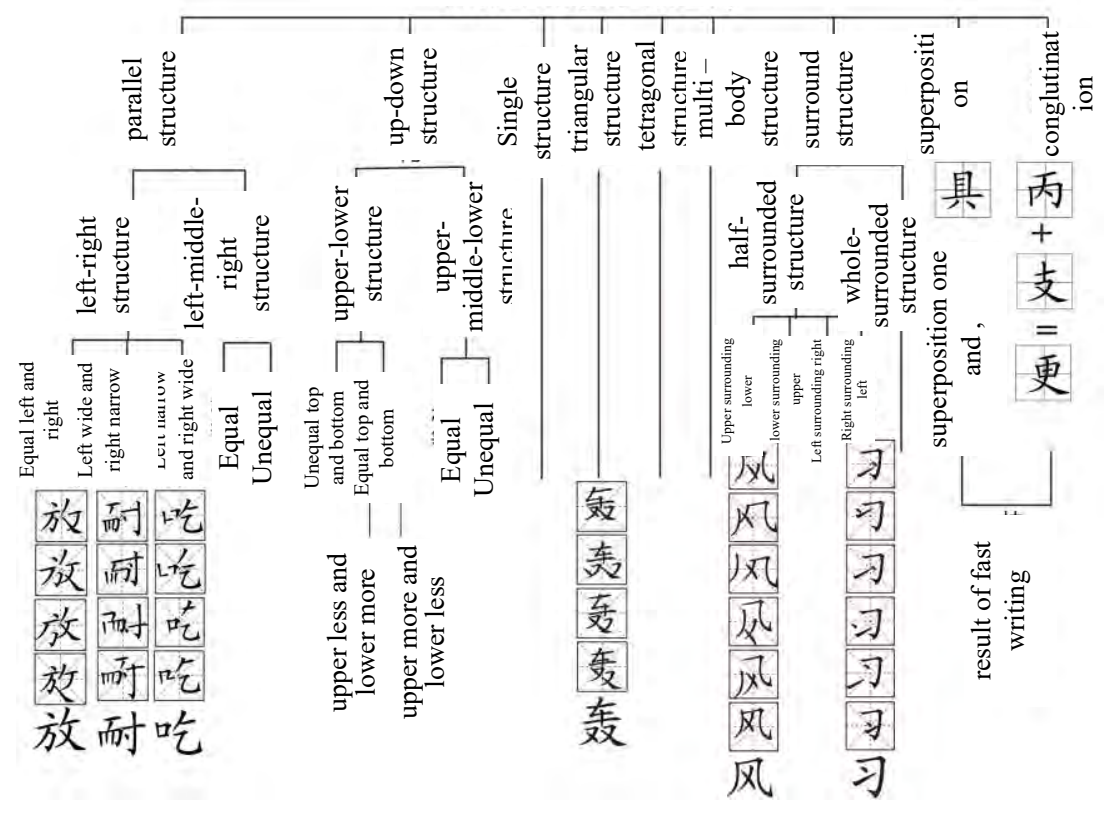

Fig. 4. Various combination modes of Chinese characters in deconstruction.

\section{CHARACTERISTICS ANALYSIS OF CHINESE FonTS AFTER DECONSTRUCTION}

\section{A. Center of Gravity}

By putting together some deconstructed fonts for comparison and analysis, we find the overall gravity is uncertain. The fonts deconstructed according to the gravity transfer method are naturally biased and unstable, while the fonts deconstructed by the methods of deconstruction and construction, and of configuration level and combination mode, are uncertain, which may be biased or may be in the center or in the vertical line of the structure. If not making experiment and designing the fonts according to these methods, it's easy to fall into the mindset of font skew and unstable gravity center, but in fact, the center of gravity is uncertain in the process of deconstruction. As shown in "Fig. 5 ", the author selects several fonts deconstructed by different methods, and marks the center of gravity after they are shaped. These fonts have a single structure, a surrounding structure of right encircling left and of lower encircling upper, a left-right structure, and a multi-body structure. For example, the deconstructed fonts such as "习(xi)" and "风 (feng)" have a unstable center of gravity, with offsets in different directions, for which these font styles are all performed by the method of gravity center offset during the deconstruction process; while the word of “放 (fang)" has a parallel structure of equal left and right, and its several deconstructed fonts are obtained by the combination mode of deconstructing font, that is, changing the font style of left wide and right narrow or left narrow and right wide, and in this process, their strokes are moved, but when the spatial structure is reorganized, it is not possible to make too much change of motion — such as loosening the stroke relationship in parallel positions, to ensure the basic recognition of the font. Therefore, it creates conditions for the center of gravity not to change. By summing up the above, the gravity center of deconstructed fonts is multi-form and uncertain.

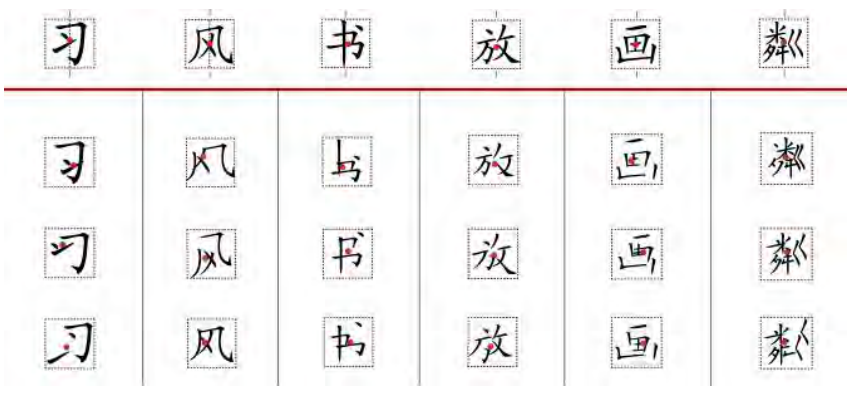

Fig. 5. Contrast of the center of gravity of the deconstructed font.

\section{B. Spatial Structure}

In order to study the spatial structure changes after the deconstruction of the regular script characters, the author extracted a part of the fonts for rational and scientific analysis. Deconstructed fonts reconstruct after the original strokes are broken up. The strokes are arranged without regularity and order, which destroys the standard spatial structure relationship, thus giving a broken, incomplete and messy visual impression. The analysis and summary are from the visual sense. In order to make it more convincing, the spatial relationship of the fonts before and after deconstruction is marked with "a circle". From "Fig. 6", we can see the red font on the left column, which is the standard printed regular script. The circle distribution is relatively uniform, and the circle represents the spatial relationship among strokes, which indicates the uniform distribution of the strokes of the original font. The black font on the right is the font form after deconstruction. By observing the change of the circles, we can see that the size difference is relatively 
large. In other words, the spatial relationship among strokes is uneven and unstable. In order to see this more clearly, the author deletes all the fonts, leaving only the circles representing the structure among the strokes, and makes the mathematical judgment of the ratio of the largest circle to the smallest circle (maximum space to minimum space), as shown in "Fig. 7". The data shows that the space ratio of the standard regular script in the left side is between 1:1.6 and $1: 3.64$, while the spatial ratio of the deconstructed font on the right is between 1:3.6 and 1:9.91. Obviously, there is a big difference between the spatial structure size of deconstructed font and that of standard regular script, which forms a new spatial structure order of non-order, giving people a conflicting visual experience and establishing a new aesthetic standard.
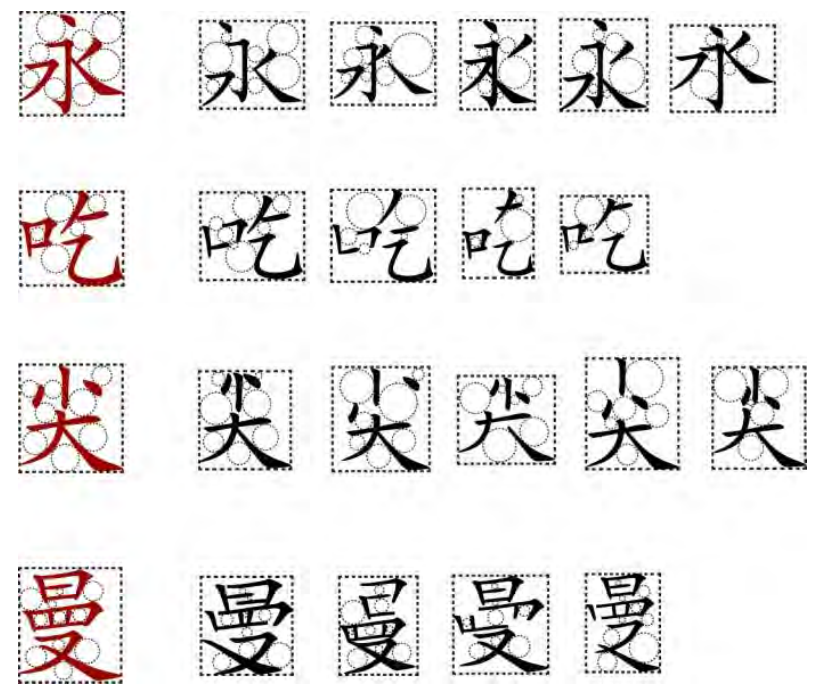

Fig. 6. Comparison of the spatial structure of standard regular script and deconstructed font.

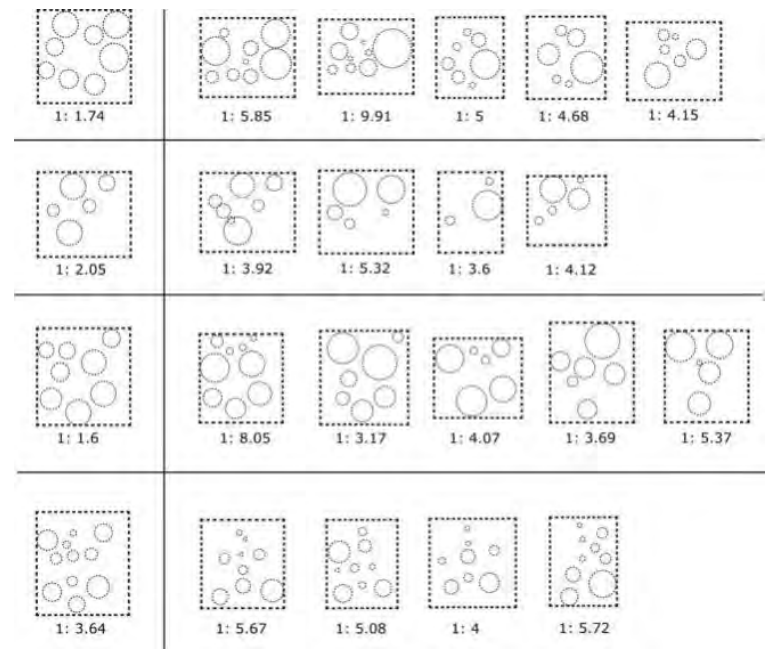

Fig. 7. Spatial structure ratios of standard regular script and deconstructed font.

In addition, it can be seen from the size change of the boxes in the figure that uses different deconstructed forms for same character and we can get font structures of different size and different length. Then, the same font in same type size may also vary in size and length in the font library. Therefore, the deconstructed font breaks the characteristic of balance beauty in standard Chinese character. Most of the fonts are irregular geometric shapes after the reorganization of the strokes. Such shape features determine that deconstructed fonts are not suitable for use in body text, and is more suitable for use in the titles of media such as exhibitions, posters, books, magazines, and web pages.(See "Fig. 8"

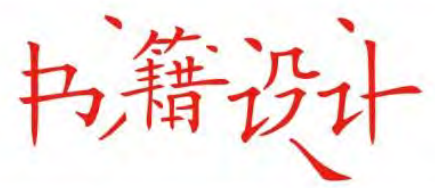

$$
\text { I B }
$$

Fig. 8. Application of deconstructed font in title.

\section{CONCLUSION}

The new font style created by the deconstructive method provides a new perspective for modern Chinese character design, which has practical application value. The information age calls for the continuous innovation and development of fonts, and the innovation of Chinese characters is not only to ensure the rigorous standardization of Chinese characters, but also to provide sufficient development space for innovative design. Our designers have the responsibility to create more personalized fonts with a rigorous attitude and push to market in order to meet the aesthetic consciousness and appreciation habits of people in the new era.

\section{REFERENCES}

[1] Lei Dehou (German): "All Things - Modularization and Large-scale Production in Chinese Art", Beijing, Sanlian Bookstore, 2005. (in Chinese)

[2] Li Mingjun: "The Historical Illustration of Chinese Characters of Fine Art ", Beijing, People's Fine Arts Publishing House, 1997. (in Chinese)

[3] Wang Ning: Lecture on Chinese Character Configuration, Shanghai, Shanghai Century Publishing Group Shanghai Education Press, 2002. (in Chinese)

[4] "Visual Design of Art Words and its Ambiguity", Liu Zanai, in "Decoration", 1986, 2nd issues. (in Chinese)

[5] Wu Lieyan: "Deconstruction Design", Nanjing, Jiangsu Fine Arts Publishing House, August 2001. (in Chinese)

[6] Huang Qihong: "Derrida's Theory on Art", Jilin, Jilin Fine Arts Publishing House, December 2007. (in Chinese) 